\title{
Cohort differences in exercise adherence among primary care patients referred for mental health versus physical health conditions
}

\author{
Patrick Tobi ${ }^{1}$, Philip Kemp ${ }^{2}$ and Elena Schmidt ${ }^{3}$ \\ ${ }^{1}$ Principal Research Fellow, Institute for Health and Human Development, University of East London, Stratford, UK \\ ${ }^{2}$ Visiting Fellow, Faculty of Health and Social Care, London South Bank University, London, UK \\ ${ }^{3}$ Director of Strategic Programme Development, Evidence and Research, Sightsavers, Haywards Heath, West Sussex, UK
}

\begin{abstract}
Aim: To compare the characteristics of mental health and physical health participants attending an exercise referral scheme (ERS) and investigate associations with their adherence to exercise. Background: While people referred to an ERS with a mental health diagnosis have similar initial rates of uptake as physical health participants, they are more likely to drop out. Comparisons of the groups to understand their differences and how these might impact on their adherence have been limited by the typically low numbers of mental health referrals in many schemes. Methods: Retrospective analysis of a participant cohort. Data were extracted on all participants enrolled over a 12- month period ( $\mathrm{n}=701$ ) and included measurements at baseline, mid-point (13 weeks) and end of programme (20-26 weeks). Differences were explored between the mental health $(n=141)$ and physical health $(n=560)$ subcohorts, and between adherers and non-adherers in each group. Binomial logistic regression estimated the effect of group-level factors associated with adherence. Findings: Mental health referrals were more likely to be younger, White and unemployed, and had a lower mean body mass index and lower proportion of participants with high blood pressure. They were also more likely to drop out. While occupation was associated with exercise adherence among the physical health group, no predictive factors were identified in the mental health group. Conclusion: Participants referred for mental health disorders are more likely to drop out of exercise referral schemes than those with physical health problems. While no factors were found to be predictive of their exercise adherence, an understanding of their distinguishing characteristics and attendance behaviour can guide in making better referral decisions concerning them and planning more appropriately tailored support.
\end{abstract}

Key words: exercise referral; exercise adherence; mental health; physical health

Received 21 September 2016; revised 24 February 2017; accepted 20 March 2017; first published online 27 June 2017

\section{Introduction}

Regular exercise has a range of recognised benefits for physical health and mental well-being, both in terms of treating and preventing ill health. Government guidelines for adults recommend at

Correspondence to: Patrick Tobi, Principal Research Fellow, Institute for Health and Human Development, University of East London, Stratford, UK. Email: p.tobi@uel.ac.uk least $150 \mathrm{~min}$ of moderate intensity activity each week (Department of Health, 2011), yet, according to the 2012 Health Survey for England report - the latest report with a specific chapter on physical activity - only $66 \%$ of men and $56 \%$ of women achieved these levels (Scholes and Mindell, 2013). As a result, physical activity has continued to remain high on the government's public health agenda. For example, tackling physical inactivity is a key strategy for delivering on many of the seven

(C) Cambridge University Press 2017 
priorities identified by Public Health England to tackle the behaviours that increase the risk of poor mental and physical health (Varney et al., 2014). A legacy commitment from the London 2012 Olympic and Paralympic Games is to increase the number of adults taking at least $150 \mathrm{~min}$ of physical activity a week and to reduce the number taking $<30 \mathrm{~min} /$ week, year on year (Her Majesty's Government, 2014).

Exercise referral schemes are one of the main routes through which exercise therapy is delivered in primary care in the United Kingdom [National Institute for Health and Clinical Excellence (NICE), 2006a]. There are different models of practice but they typically involve a primary care or allied health professional referring a patient to a dedicated exercise professional who designs a formal exercise programme tailored to the patient's fitness level, motivation, and goals. The programme usually takes place within a leisure facility or gym over 10-12 weeks and is offered at a subsidised cost to encourage participation (Department of Health, 2001; BHF National Centre, 2009). Structured exercise programmes of this nature are appealing because of their capacity to offer safe and tailored physical activity in a supervised setting and because adherence can be more easily demonstrated than with lifestyle interventions (Richardson et al., 2005).

Notwithstanding their popularity, adherence rates on the schemes are typically low and many participants fail to complete the scheduled sessions (Williams et al., 2007). This is particularly true of people referred with a mental health diagnosis, and research shows that while they may have similar initial rates of uptake as other participants, they are more likely to drop out (Crone et al., 2008). Several studies have sought to investigate a range of issues in this group such as their initial uptake, subsequent participation and adherence, motivation to attend, perceptions about the schemes, and barriers to participation (Crone et al., 2005; Crone and Guy, 2008; Myron et al., 2009). While these have provided useful insights, the reasons for the high drop out rate still need to be better understood (Crone et al., 2008).

A common feature in many exercise referrals schemes is that people with mental health problems often comprise a low proportion of those who participate. This can present a challenge for robust statistical investigation and may partly explain why not much is known about whether and how people referred for exercise on account of mental health problems differ from those referred for physical health problems. Of particular interest is whether any identified differences might advance understanding of the differing rates of adherence in the two groups; the main research question this study aims to address.

We report the results of a retrospective registerbased analysis of Healthwise, an exercise referral scheme in the inner city London borough of Greenwich. While the scheme participants were mostly people with a range of physical health conditions, a relatively high proportion, $20 \%$, were referred with a primary mental health diagnosis. Participation and adherence rates were high compared with similar schemes: 1089 participants were enrolled between January and December 2007, adherence was 58\% at 13 weeks (the average duration of typical schemes), and $45 \%$ completed the full course duration of 20-26 weeks of regular exercise. This contrasts with a pooled adherence level of $49 \%$ reported from a systematic review of studies (Pavey et al., 2012). Main results from the whole cohort have been reported elsewhere (Tobi et al., 2012). This paper illuminates the distinctive socio-demographic features of mental health participants, comparing them with those referred for physical health conditions, and exploring associations with exercise adherence in both groups.

\section{Method}

\section{Study setting}

Healthwise was formed in 2005 as a partnership between the local council, local health authority, and a commercial provider of leisure services. It was aimed at adult residents with, and at risk of, physical and mental health illnesses for whom exercise therapy could be beneficial in terms of weight loss, improved fitness, and reduction in symptoms or risk. It also targeted people from a Black and Ethnic Minority (BME) background, who formed an estimated $29 \%$ of the borough's population and, as a group, experienced worse health inequalities (Greenwich Teaching Primary Care Trust, 2008).

Referrals were made from general practice (GP) surgeries in the borough to a leisure facility where participants were enrolled on a supervised, tailored exercise programme over a period of 20-26 weeks. This duration was double the length 
Table 1 Clinical referral categories of participants

\begin{tabular}{|c|c|c|c|}
\hline Referral category & Medical conditions within category & $n$ & $\%$ \\
\hline Metabolic & Diabetes, hyperlipidaemia, obesity, hyperthyroidism, hypothyroidism & 228 & 33 \\
\hline Orthopaedic & Arthritis, back pain, osteoporosis, fibromyalgia, other bone/musculoskeletal conditions & 164 & 23 \\
\hline Mental health & Depression, anxiety, stress, other mental disorders & 141 & 20 \\
\hline Cardiovascular & $\begin{array}{l}\text { Myocardial infarction, coronary artery bypass graft surgery and coronary angioplasty, angina } \\
\text { and silent ischaemia, atrial fibrillation, chronic heart failure, peripheral arterial disease, } \\
\text { hypertension }\end{array}$ & 111 & 16 \\
\hline Respiratory & Chronic obstructive pulmonary disease, asthma & 34 & \\
\hline $\begin{array}{l}\text { Neuromuscular/ } \\
\text { sensory/others }\end{array}$ & $\begin{array}{l}\text { Stroke, spinal cord disabilities, multiple sclerosis, cerebral palsy, Parkinson's disease, Down's } \\
\text { syndrome, brain injury, deaf and hard of hearing, visual impairment, cancer, haemangioma, } \\
\text { epilepsy, HIV, hepatitis C, renal disease }\end{array}$ & 23 & \\
\hline
\end{tabular}

of most other schemes which generally last about 10-12 weeks (BHF National Centre, 2009). The referrals represented a wide range of clinical conditions distributed across six broad categories (Table 1). Participants attended motivational and educational classes and had access to a range of courses including group exercise, healthy walks, gym-based sessions, water workouts, and swimming. Physical and physiological assessments were performed at enrolment and at seven, 13 and 20-26 weeks.

\section{Programme data}

All records entered in the scheme register between 1 January and 31 December $2007(n=1089)$ were extracted. Using a 12-month time frame cancelled out the effect of seasonal variations in exercise levels (Ma et al., 2006; Tucker and Gilliland, 2007). We excluded 322 cases that were still in progress at the time and another 66 cases that were ineligible for other reasons (did not qualify, did not start, missing data on key variables). The final cohort $(n=701)$ was separated into mental health $(n=141)$ and physical health $(n=560)$ groups. The latter was composed of all the non-mental health categories described in Table 1.

\section{Primary outcome}

Adherence was the main outcome measure for this study and determined by both the frequency and duration of attendance. The justification for this definition is detailed in Tobi et al. (2012). We defined adherers as participants who attended at least $80 \%$ of their scheduled sessions and had records of two progress assessments at the midpoint (13th week) and end (20-26th week) of the programme. Non-adherers were those who attended
$<80 \%$ of sessions and/or failed to undergo any of the two assessments. The $80 \%$ cut-off point was based on participant data analysed in an earlier study (Gidlow et al., 2007).

\section{Process measures}

Explanatory variables were selected from the participant register and included age, gender, ethnicity, occupational group, medical reason for referral, blood pressure (BP), height, and weight. Body mass index (BMI) at enrolment was computed from height and weight measurements. Although BP and BMI were measured as continuous data, we also categorised them to reflect recognised clinical cut-off points. Accordingly, BP at enrolment was classified as normal if the systolic (SBP) and diastolic (DBP) readings were $<140$ and $<90 \mathrm{mmHg}$, respectively, or high if either the SBP was $\geqslant 140$ or DBP $\geqslant 90 \mathrm{mmHg}$ (NICE, 2006b). BMI was categorised as healthy $\left(18.5-24.9 \mathrm{~kg} / \mathrm{m}^{2}\right)$, overweight $\left(25.0-29.9 \mathrm{~kg} / \mathrm{m}^{2}\right)$, or obese $\left(\geqslant 30 \mathrm{~kg} / \mathrm{m}^{2}\right)$ in line with national guidance (NICE, 2005). Each participant was assigned an Index of Multiple Deprivation (IMD) score based on their postcode address. The IMD is a composite measure of relative deprivation in small areas of England called Lower Super Output Areas and uses the scores for the areas to rank them from 1 (most deprived) to 32482 (least deprived) (Office of the Deputy Prime Minister, 2004).

\section{Data analysis}

After exploratory analysis to ascertain completeness of the data and normality of the distribution, analysis was done in three steps. Bivariable analysis was carried out to first explore differences between the mental health and physical health sub-cohorts (step 1) and then between adherers and non-adherers 
within each group (step 2). We used the $\chi^{2}$ test to compare categorical variables and the $t$ test for continuous variables. In the final step, binomial logistic regression was used to estimate the effect of factors associated with exercise adherence at group level. Significant multicollinearity and interactions were excluded before fitting all covariates that demonstrated association with adherence at the $20 \%$ level into regression models. The Hosmer-Lemeshow test was used to check overall model fit. Principal analyses were presented with three key statistical items - point estimate, confidence interval, and $P$-value - in line with reporting guidelines (Peacock and Kerry, 2006;
Vandenbroucke et al., 2007). All analysis was done with PASW Statistics 18.

\section{Results}

\section{Characteristics of participants}

Table 2 presents the socio-demographic characteristics of participants with physical and mental health conditions. Compared to those with physical health conditions, mental health referrals were more likely to be younger (41.3 versus 47.4 years, $P<0.001)$ and white (71 versus $61 \%, P=0.03$ ).

Table 2 Comparative profile of participants

\begin{tabular}{llll}
\hline Characteristics & Mental health & Physical health & $P$ \\
\hline Mean age (SD) (years) & $41.3(11.8)$ & $47.4(14.2)$ & $<0.001^{\mathrm{a}}$ \\
Women (\%) & 63.1 & 58.6 & $0.32^{\mathrm{b}}$ \\
Black/Minority Ethnicity (\%) & 29.0 & 38.9 & $0.03^{\mathrm{b}}$ \\
Occupational group (\%) & & & $<0.001^{\mathrm{b}}$ \\
$\quad$ Unemployed & 60.8 & 32.8 & \\
Retired & 9.2 & 20.4 & \\
Unskilled/partly skilled & 3.1 & 10.5 & \\
Skilled & 16.9 & 24.7 & $0.97^{\mathrm{b}}$ \\
Managerial/professional & 10.0 & 31.7 & $0.01^{\mathrm{a}}$ \\
Mean IMD score (SD) & $35.7(12.2)$ & $32.6(12.3)$ & $0.01^{\mathrm{b}}$ \\
Mean baseline BMI (SD) & $30.8(6.1)$ & 44.7 & $0.04^{\mathrm{b}}$ \\
Elevated baseline BP (\%) & 32.6 & 47.0 & \\
Adherence to exercise (\%) & 37.6 & & \\
\hline
\end{tabular}

$\mathrm{IMD}=$ Index of Multiple Deprivation; $\mathrm{BMI}=$ body mass index; $\mathrm{BP}=$ blood pressure. ${ }^{\mathrm{a}} t$ Test.

b $\chi^{2}$ Test.

Table 3 Group-level factors associated with exercise adherence

\begin{tabular}{|c|c|c|c|c|c|c|}
\hline \multirow[b]{2}{*}{ Covariate } & \multicolumn{3}{|c|}{ Mental health } & \multicolumn{3}{|c|}{ Physical health } \\
\hline & Adherers & Non-adherers & $P$ & Adherers & Non-adherers & $P$ \\
\hline Mean age (SD) (years) & $43.5(11.1)$ & $40.1(12.1)$ & $0.10^{\mathrm{a}}$ & $49.4(13.3)$ & $45.7(13.3)$ & $0.002^{a}$ \\
\hline Women (\%) & 67.9 & 60.2 & $0.36^{\mathrm{b}}$ & 59.0 & 58.2 & $0.85^{\mathrm{b}}$ \\
\hline Black/Minority Ethnicity (\%) & 37.3 & 24.1 & $0.10^{\mathrm{b}}$ & 36.8 & 40.3 & $0.41^{\mathrm{b}}$ \\
\hline Occupational group (\%) & & & $0.51^{\mathrm{b}}$ & & & $0.01^{b}$ \\
\hline Unemployed & 64.6 & 58.5 & & 26.8 & 38.5 & \\
\hline Retired & 12.5 & 7.3 & & 25.6 & 15.4 & \\
\hline Unskilled/partly skilled & 4.2 & 2.4 & & 11.4 & 9.6 & \\
\hline Skilled & 12.5 & 19.5 & & 26.4 & 23.1 & \\
\hline Managerial/professional & 6.3 & 12.2 & & 19.8 & 13.5 & \\
\hline Mean IMD score (SD) & $35.9(11.8)$ & $35.5(12.5)$ & $0.86^{\mathrm{a}}$ & $35.2(12.4)$ & $36.0(12.2)$ & $0.49^{a}$ \\
\hline Mean baseline BMI (SD) & $31.1(5.4)$ & $30.5(6.5)$ & $0.60^{\mathrm{a}}$ & $32.1(7.1)$ & $32.6(7.3)$ & $0.35^{a}$ \\
\hline Elevated baseline BP (\%) & 43.4 & 26.1 & $0.03^{b}$ & 44.0 & 45.0 & $0.82^{\mathrm{b}}$ \\
\hline
\end{tabular}

IMD = Index of Multiple Deprivation; BMI = body mass index; $\mathrm{BP}=$ blood pressure.

${ }^{\mathrm{a}} t$ Test.

b $\chi^{2}$ Test.

Primary Health Care Research \& Development 2017; 18: 463-471 
Table 4 Adjusted odds ratios (OR) for exercise adherence

\begin{tabular}{|c|c|c|c|c|c|c|}
\hline \multirow[t]{2}{*}{ Covariate } & \multicolumn{3}{|c|}{ Mental health model } & \multicolumn{3}{|c|}{ Physical health model } \\
\hline & OR & $95 \% \mathrm{Cl}$ & $P$ & OR & $95 \% \mathrm{Cl}$ & $P$ \\
\hline Age (continuous) & 1.01 & $0.98-1.05$ & 0.49 & 1.01 & $0.99-1.03$ & 0.23 \\
\hline \multicolumn{7}{|l|}{ Ethnicity } \\
\hline Black Minority & 1.82 & $0.85-3.89$ & 0.12 & & & \\
\hline \multicolumn{7}{|l|}{ Blood pressure } \\
\hline Normotensive & 1.00 & Ref. & & & & \\
\hline Hypertensive & 1.70 & $0.75-3.85$ & 0.20 & & & \\
\hline Occupation & & & & & & 0.17 \\
\hline Unemployed & & & & 1.0 & Ref. & \\
\hline Retired & & & & 1.85 & $1.00-3.43$ & 0.05 \\
\hline Unskilled/partly skilled & & & & 1.58 & $0.84-2.96$ & 0.16 \\
\hline Skilled & & & & 1.58 & $0.98-2.53$ & 0.06 \\
\hline Managerial/professional & & & & 1.01 & $0.55-1.89$ & 0.96 \\
\hline
\end{tabular}

$\mathrm{Cl}=$ confidence interval; Ref. $=$ reference category .

The profile of occupational categories in the groups also differed significantly $(P<0.001)$. Proportionally twice as many mental health than physical health participants were unemployed (61 versus $33 \%$ ) while the ratio was reversed among those who were retired (9 versus $20 \%$ ). Only in managerial/professional roles was there some parity (10 versus $12 \%$ ).

The two clinical measures of health state captured in the data set were BMI and BP. Although mean BMI was significantly lower in mental health referrals $\left(30.8\right.$ versus $\left.32.5 \mathrm{~kg} / \mathrm{m}^{2}, P=0.01\right)$, the average value in both groups fell within the range of obesity. Mental health participants had a lower proportion of people with high BP (33 versus $45 \%, P=0.01)$, but were also less likely to maintain attendance over the duration of the scheme (38 versus $47 \%, P=0.04$ ).

\section{Factors associated with exercise adherence}

The same variables in Table 2 were further used to explore adherence-related factors within each group (Table 3). Among mental health referrals, exercise adherence was more likely in participants with an elevated baseline BP (43 versus $26 \%, P=0.03$ ). With physical health participants, those more likely to adhere were older ( 49 versus 46 years, $P=0.002$ ), unemployed (27 versus $39 \%$ ), or retired (26 versus $15 \%, P=0.01)$.

Table 4 shows the result of regression models for the two groups. We used associations with adherence at $P<0.20$ significance level to select covariates to build the models. Accordingly, age, ethnicity, and BP were fitted to the mental health model, and age and occupation to the physical health model. While no covariates were found to be independently predictive in the mental health group, occupation was associated with adherence in the physical health group where the odds of retired participants adhering were almost twice those of unemployed participants after adjusting for age $(\mathrm{OR}=1.85 ; \mathrm{CI}=1.00-3.43 ; P=0.05)$.

\section{Discussion}

Although there has been a sustained growth in the number of exercise referral schemes in the United Kingdom since 1990s, inadequate monitoring and participating profiling has limited efforts to better understand who these schemes are accessible to and suitable for. Consequently, the importance of more appropriate targeting at the point of referral as a means of improving participation rates has been highlighted (Department of Health, 2001; James et al., 2008; BHF National Centre, 2009). The need to understand these factors is especially important among groups with lower participation rates for whom the schemes may be ill designed or even inappropriate. In the case of mental health participants, the growing use of exercise therapy in the mental health service, including in people with serious mental illness (Ellis et al., 2007), has given 
added impetus to efforts to develop more evidence-based practice.

Several factors may explain the comparatively lower numbers of mental health referrals. One reason may be that the schemes have traditionally been designed to deal with physical health conditions (Directorate of Health Promotion, 2003) and so naturally tend to recruit participants with these problems. Questions have also been raised about whether health professionals refer inappropriately. Health professionals are known to make subjective judgements about which patients might be most motivated to adhere (Din et al., 2015). Furthermore, their referral behaviour may be influenced by perceptions about the strength of the evidence, notwithstanding that associations between exercise and mental well-being have been repeatedly recognised for many years (Stathopoulou et al., 2006). Whatever the reason, low numbers of mental health participants has meant that few studies have been able to undertake any robust analysis of this group from a quantitative perspective. Our participant cohort was different in this respect, having a relatively high proportion $(20 \%)$ of mental health referrals.

Our analysis found that participants referred with a primary mental health condition were more likely to drop out of the ERS, consistent with findings elsewhere (Dugdill et al., 2005; Crone et al., 2008). Older age and poorer physical health are factors typically associated with lower levels of physical activity in the general population (Craig et al., 2009) and, presumably, adherence. But the mental health cohort were comparatively younger and, given their lower mean BMI and lower proportion of people with elevated BP, arguably in better health than the physical health group. So, even though it might reasonably be inferred that they would be more likely to maintain attendance, that was not the case. The sole factor that was significantly associated with adherence among the mental health group was an elevated BP at the time of enrolment, which may possibly have acted as a motivation to adhere to the exercise regime. However, when age and ethnicity were taken into account, the association was no longer apparent. BMI, the other clinical measure of health state, did not show any relationship.

The proportion of BME participants in the mental health group was significantly lower than in the physical health group (although similar to that of the general population in the borough). However, without information about rates of mental ill health among different ethnic groups in the primary care patient pool from which participants were referred to the ERS, it is difficult to infer any significance to this. But studies of mental health services for BME people highlight variations in the referral practices of GPs and their perceptions about who might benefit from the scheme. This can manifest in different ways such as the tendency for services to medicate BME patients in preference to other forms of therapy or a lack of cultural competence making misdiagnosis and inappropriate treatment more likely (Bhui et al., 2007; Fountain and Hicks, 2010). Ethnic disparities in health care access, both in mental health and other service areas, continue to remain a significant scientific and policy challenge and focus of health services research.

Although information about levels of motivation at enrolment was not available to us, lower levels of adherence among individuals with mental health problems may relate to the nature of many mental health problems themselves being associated with poor motivation. People with depression will experience lack of energy and no longer experience the enjoyment they once did while exercising or undertaking a range of daily activities (Seime and Vickers, 2006). This is particularly the case for those with long-term conditions. They often need a more supportive or even assertive approach to adhere to social interventions aimed at improving their quality of life. However, people with anxiety-related disorders may seek to avoid social situations, such as the group-based activities within the programme, which might exacerbate their anxiety. Ironically, these group activities are supposed to serve as a means of providing the added social support these participants are known to require.

Attention needs to be drawn to the policy context in which the programme was implemented and its potential influence on outcomes. Few ERS studies address the influence of the wider context of service delivery to explain adherence outcomes, but this can be important. Healthwise was developed as part of a broader strategic agenda to tackle local health inequalities and the referral policy prioritised BME participants who for the most part suffered worse health outcomes. If any targeted support was given to them in the programme, then it could 
contribute to the higher adherence rates seen in the physical health group where there was a comparatively higher proportion of BME participants.

Finally, our analysis relied exclusively on the routinely collected demographic and physiological data, but it is difficult to reach any firm conclusions about the absence of variation in attendance in the mental health referral group, solely on this basis. Participation in activities is a complex behaviour that, useful as it is, quantitative data can only present a partial picture of. Evidence from qualitative research plays a vital role in describing the processes that influence people's physical activity behaviour (Mckenna and Mutrie, 2003). Qualitative research is particularly useful in understanding the experiences, psychological and social influences, and contexts that enable, support, and sustain physical behaviour, or otherwise constrain it. Qualitative ERS studies have been conducted, including research in relation to mental health participants, but the still limited understanding suggests a place for mixed method studies that combine both quantitative and qualitative perspectives. Physical activity research papers based on mixed methods are increasingly appearing in the literature as researchers recognise the added value they can bring (Thomas et al., 2015).

The study had several limitations. Although we used a 12-month time frame in an attempt to adjust for seasonality effect, this may not have been sufficient. It is possible, for instance, that people with mental health conditions are more commonly referred during the winter months when these conditions are often exacerbated. Reliable data on the time of referral to the ERS were not available. The physical health of individuals with mental health illness is often poor, with many being overweight or obese, having hypertension, diabetes, or dyslipidaemia, and at significant risk of developing cardiovascular disease or other comorbidities. Similarly, people with physical health conditions may also experience depression, stress, and other common mental disorders. By disaggregating the groups only on the basis of their primary medical diagnosis without considering comorbidities, this confounder was not accounted for. We were also not able to verify the accuracy of the referral diagnosis. Another problem was the limited participant information available. Factors such as levels of physical activity and motivation at the start of the programme are known to influence adherence to exercise, and several studies (Bock et al., 1997; Marshall and Biddle, 2001) have applied models of motivational readiness for behavioural change to explain the process of exercise adoption and maintenance. Finally, given that Healthwise operated in a primary care setting, all the mental health participants were diagnosed with common mental disorders (anxiety, depression, stress). The results will not necessarily be the same for people with serious mental illness.

\section{Conclusion}

This retrospective study of an exercise referral scheme highlights the differences between participants with a primary mental health diagnosis and those with physical health ones. It provides useful insights, previously unavailable, into the differing rates of adherence in the groups. The information can guide service commissioners and providers in developing strategies to maintain exercise tailored to the unique characteristics of the different participant groups.

\section{Acknowledgements}

The data analysed in this study were taken from an evaluation of Healthwise funded by Greenwich Leisure Limited (GLL) and NHS Greenwich.

\section{References}

BHF National Centre. 2009: A toolkit for the design, implementation and evaluation of exercise referral schemes. Guidance for referring health professionals, exercise referral professionals and exercise referral scheme commissioners, Draft Consultation Report. Loughborough: Loughborough University, British Heart Foundation National Centre for Physical Activity and Health.

Bhui, K., Warfa, N., Edonya, P., McKenzie, K. and Bhugra, D. 2007: Cultural competence in mental health care: a review of model evaluations. BMC Health Services Research 7, 15.

Bock, B.C., Albrecht, A.E., Traficante, R.M., Clark, M.M., Pinto, B.M., Tilkemeier, P. and Marcus, B.H. 1997: Predictors of exercise adherence following participation in a cardiac rehabilitation program. International Journal of Behavioral Medicine 4, 60-75.

Craig, R., Mindell, J. and Hirani, V. 2009: The Health Survey for England 2008: Physical activity and fitness. London: NHS Information Centre for Health and Social Care.

Crone, D. and Guy, H. 2008: 'I know it is only exercise, but to me it is something that keeps me going': a qualitative 
approach to understanding mental health service users' experiences of sports therapy. International Journal of Mental Health Nursing 17, 197-207.

Crone, D., Johnston, L.H., Gidlow, C., Henley, C. and James, D.V. 2008: Uptake and participation in physical activity referral schemes in the UK: an investigation of patients referred with mental health problems. Issues in Mental Health Nursing 29, 1088-97.

Crone, D., Smith, A. and Gough, B. 2005: 'I feel totally at one, totally alive and totally happy': a psycho-social explanation of the physical activity and mental health relationship. Health Education Research 20, 600-11.

Department of Health. 2001: Exercise referral systems: a national quality assurance framework. London: Department of Health.

Department of Health. 2011: Start active, stay active: a report on physical activity in the UK. London: Department of Health.

Din, N.U., Moore, G.F., Murphy, S., Wilkinson, C. and Williams, N.H. 2015: Health professionals' perspectives on exercise referral and physical activity promotion in primary care: findings from a process evaluation of the National Exercise Referral Scheme in Wales. Health Education Journal 74, 743-57.

Directorate of Health Promotion. 2003: Lyme Brook and Newcastle borough council exercise referral pilot; evaluation report. Newcastle: Staffordshire Moorlands, North and South Stoke PCTs.

Dugdill, L., Graham, R.C. and McNair, F. 2005: Exercise referral: the public health panacea for physical activity promotion? A critical perspective of exercise referral schemes; their development and evaluation. Ergonomics 48, 1390-410.

Ellis, N., Crone, D., Davey, R. and Grogan, S. 2007: Exercise interventions as an adjunct therapy for psychosis: a critical review. British Journal of Clinical Psychology 46, 95-111.

Fountain, J. and Hicks, J. 2010: Delivering race equality in mental health care: report on the findings and outcomes of the community engagement programme 2005-2008. Preston: International School for Communities, Rights and Inclusion (ISCRI), University of Central Lancashire.

Gidlow, C., Johnston, L.H., Crone, D., Morris, C., Smith, A., Foster, C. and James, D.V. 2007: Socio-demographic patterning of referral, uptake and attendance in Physical Activity Referral Schemes. Journal of Public Health (Oxford England) 29, 107-13.

Greenwich Teaching Primary Care Trust. 2008: Building health into the future: a report on health inequalities in Greenwich, The Annual Report of the Director of Public Health 2008. London: Directorate of Public Health, Greenwich TPCT.

Her Majesty's Government. 2014. Moving more, living more: the physical activity Olympic and Paralympic legacy for the nation. London: HMG.

James, D.V., Johnston, L.H., Crone, D., Sidford, A.H., Gidlow, C., Morris, C. and Foster, C. 2008: Factors associated with physical activity referral uptake and participation. Journal of Sports Sciences 26, 217-4.

Primary Health Care Research \& Development 2017; 18: 463-471
Ma, Y., Olendzki, B.C., Li, W., Hafner, A.R., Chiriboga, D., Hebert, J.R., Campbell, M., Sarnie, M. and Ockene, I.S. 2006: Seasonal variation in food intake, physical activity, and body weight in a predominantly overweight population. European Journal of Clinical Nutrition 60, 519-28.

Marshall, S.J. and Biddle, S.J. 2001: The transtheoretical model of behavior change: a meta-analysis of applications to physical activity and exercise. Annals of Behavioral Medicine 23, 229-46.

McKenna, J. and Mutrie, N. 2003: Emphasizing quality in qualitative papers. Journal of Sports Science 21, 955-58.

Myron, R., Street, C., Robotham, D. and James, K. 2009. Moving on up. London: Mental Health Foundation.

National Institute for Health and Clinical Excellence (NICE). 2005: Obesity: guidance on the prevention, identification, assessment and management of overweight and obesity in adults and children. NICE clinical guideline 43. London: NICE.

National Institute for Health and Clinical Excellence (NICE). 2006a: Four commonly used methods to increase physical activity: brief interventions in primary care, exercise referral schemes, pedometers and community-based exercise programmes for walking and cycling. Public Health Intervention Guidance No. 2. London: NICE.

National Institute for Health and Clinical Excellence (NICE). 2006b: Hypertension: management of hypertension in adults in primary care (partial update of NICE clinical guideline 18). NICE clinical guideline 34. London: NICE.

Office of the Deputy Prime Minister. 2004: The English indices of deprivation 2004. London: Stationary Office.

Pavey, T., Taylor, A., Hillsdon, M., Fox, K., Campbell, J., Foster, C., Moxham, T., Mutrie, N., Searle, J. and Taylor, R. 2012: Levels and predictors of exercise referral scheme uptake and adherence: a systematic review. Journal of Epidemiology and Community Health 66, 737-44.

Peacock, J. and Kerry, S. 2006. Presenting medical statistics from proposal to publication: a step-by-step guide. Oxford: Oxford University Press.

Richardson, C.R., Faulkner, G., McDevitt, J., Skrinar, G.S., Hutchinson, D.S. and Piette, J.D. 2005: Integrating physical activity into mental health services for persons with serious mental illness. Psychiatric Service 56, 324-31.

Scholes, S. and Mindell, J. 2013: Physical activity in adults. In Craig, R. and Mindell, J. editors, Health Survey for England 2012 Chapter 2, Volume 1. Leeds: Health and Social Care Information Centre, 1-79.

Seime, R.J. and Vickers, K.S. 2006: The challenges of treating depression with exercise: from evidence to practice. Clinical Psychology: Science and Practice 13, 194-97.

Stathopoulou, G., Powers, M.B., Berry, A.C., Smits, J.A.J. and Otto, M.W. 2006: Exercise interventions for mental health: a quantitative and qualitative review. Clinical Psychology: Science and Practice 13, 179-93.

Thomas, J.R., Silverman, S. and Nelson, J. 2015: Research methods in physical activity, seventh edition. Champaign, IL: Human Kinetics. 
Tobi, P., Estacio, E.V., Renton, A., Yu, G. and Foster, N. 2012: Who stays, who drops out? Biosocial predictors of longer term adherence in participants attending an exercise referral scheme in the UK. BMC Public Health 12, 347.

Tucker, P. and Gilliland, J. 2007: The effect of season and weather on physical activity: a systematic review. Public Health 121, 909-22.

Vandenbroucke, J.P., von Elm, E., Altman, D.G., Gøtzsche, P.C., Mulrow, C.D., Pocock, S.J., Poole, C., Schlesselman, J.J. and Egger, M., for the STROBE Initiative. 2007:
Strengthening the Reporting of Observational Studies in Epidemiology (STROBE): explanation and elaboration. PLoS Medicine 4, e297.

Varney, J., Brannan, M. and Aaltonen, G. 2014: Everybody active, every day: an evidence-based approach to physical activity. London: Public Health England.

Williams, N.H., Hendry, M., France, B., Lewis, R. and Wilkinson, C. 2007: Effectiveness of exercise-referral schemes to promote physical activity in adults: systematic review. British Journal of General Practice 57, 979-86. 